05

\title{
Туннелирование СВЧ излучения через трехслойные структуры, содержащие ферритовый слой
}

\author{
(C) С.А. Афранасьев, Д.И. Семенцов, К.В. Шарипова
}

Ульяновский государственный университет, 432970 Ульяновск, Россия

e-mail: asa_rpe@mail.ru

(Поступило в Редакцию 11 февраля 2018 г.)

Рассмотрено туннелирование СВЧ излучения через симметричную трехслойную структуру, в которой центральный слой феррита окружен двумя слоями материала с отрицательной диэлектрической проницаемостью. Для случая нормального падения проанализированы условия „идеального“ туннелирования, т.е. безотражательного прохождения излучения через структуру. Показано, что пропускательной способностью структуры можно управлять с помощью внешнего магнитного поля, намагничивающего феррит. В частотной области, соответствующей отрицательным значениям эффективной магнитной проницаемости феррита, возможно наличие широкой (порядка нескольких гигагерц) полосы пропускания, в которой туннелирование близко к идеальному.

DOI: 10.21883/JTF.2019.01.46966.61-18

\section{Введение}

Сравнительно недавно было обнаружено, что прохождение электромагнитного излучения через некоторые слоистые структуры, содержащие слои с отрицательными вещественными частями диэлектрической и/или магнитной проницаемости (ДП, МП), может быть полным, т.е. безотражательным. Этот эффект назван идеальным туннелированием (ИТ), он имеет резонансный характер, что выражается в резком возрастании при выполнении определенных условий амплитуд волновых полей на границах раздела сред [1-13]. Для наблюдения ИТ предложены разнообразные структуры со слоями, материалы которых известны как SNG (single-negative) среды. Среди них различаются $\varepsilon$-отрицательные (или ENG) среды с $\operatorname{Re} \varepsilon<0, \operatorname{Re} \mu>0$ и $\mu$-отрицательные (или $\mathrm{MNG}$ ) среды с $\operatorname{Re} \varepsilon>0, \operatorname{Re} \mu<0$. „Обычные“ среды с $\operatorname{Re} \varepsilon>0$ и $\operatorname{Re} \mu>0$ при этом обозначаются как DPS (double-positive).

Одиночные слои SNG сред малопрозрачны для электромагнитного излучения ввиду мнимости волновых чисел существующих в них волн. Однако многослойные структуры с SNG-слоями при выполнении определенных условий могут обеспечить ИТ. Для простейших структур с небольшим числом слоев условия ИТ могут быть получены аналитически. Так, в [7] получены и проанализированы условия ИТ для трехслойных структур четырех видов: ENG-MNG-ENG, ENG-DPS-MNG, ENG-DPS-ENG и DPS-ENG-DPS.

Основным недостатком большинства имеющихся работ по ИТ является то, что в них рассматриваются идеальные слоистые структуры без потерь. С практической точки зрения интересны реальные структуры с невысоким уровнем потерь, которые при выполнении условий ИТ обнаруживают туннелирование, близкое к идеальному. Перспективным представляется использова- ние в качестве MNG-слоев магнитоуправляемой среды, например СВЧ феррита [14]. МП феррита в области ферромагнитного резонанса зависит от частоты и поля и может принимать отрицательные значения в околорезонансной области.

В настоящей работе исследуются особенности туннелирования при нормальном падении плоской электромагнитной волны на структуру вида ENG-MNG-ENG. В качестве MNG-слоя используется поперечно намагниченный феррит. Анализ проводится для области частоты и поля, где его эффективная МП принимает отрицательные значения. На основе численного решения уравнения, выражающего условие ИТ рассматриваемой структуры, анализируются полевые и частотные зависимости ее пропускательной способности.

\section{Постановка задачи}

На рис. 1 изображена схема рассматриваемой трехслойной структуры, связанная с ней система координат, компоненты статического магнитного поля и волнового поля магнитоактивной ТЕ волны. Структура занимает

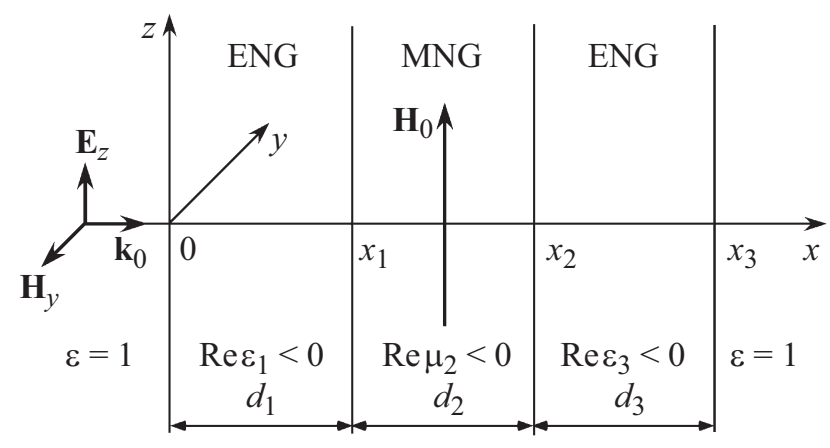

Рис. 1. Геометрия задачи. 
пространственную область $0 \leq x \leq x_{3}$ и расположена в вакууме (области $x<0$ и $x>x_{3}$ ). Ее слои предполагаются бесконечно протяженными в направлениях осей $0 y$ и $0 z$. ENG-слои 1 и 3 имеют одинаковую толщину и материальные параметры $\varepsilon_{1}<0$ и $\mu_{1}>0$. MNG-слой 2 толщиной $d_{2}$ с материальными параметрами $\varepsilon_{2}, \mu_{2}$ представляет собой феррит, намагниченный до насыщения вдоль оси $0 z$ внешним магнитным полем напряженностью $H_{0}$.

Для расчетов в качестве феррита был выбран железоиттриевый гранат с минимально возможными магнитными и электрическими потерями. Для него ширина линии магнитного резонанса и тангенс диэлектрических потерь составляют величины $\Delta H=1 \mathrm{Oe}, \operatorname{tg} \delta=2 \cdot 10^{-4}[15,16]$. Высокочастотные свойства феррита характеризуются следующим тензором МП:

$$
\begin{gathered}
\hat{\mu}=\left(\begin{array}{ccc}
\mu & -i \mu_{a} & 0 \\
i \mu_{a} & \mu & 0 \\
0 & 0 & 1
\end{array}\right) \\
\mu=\frac{\omega_{H}\left(\omega_{M}+\omega_{H}\right)-\omega^{2}}{\omega_{H}^{2}-\omega^{2}}, \quad \mu_{a}=\frac{\omega \omega_{M}}{\omega_{H}^{2}-\omega^{2}},
\end{gathered}
$$

где $\omega_{H}=\gamma H_{0}, \omega_{M}=4 \pi \gamma M_{0}, 4 \pi M_{0}=1850 \mathrm{Gs}$ - намагниченность насыщения, $\gamma$ - гиромагнитное отношение. Учет магнитных потерь проводится с помощью замены в соотношениях (1) параметра $\omega_{H}$ на $\omega_{H}+i \gamma \Delta H$ [17].

Пусть из области $x<0$ на структуру нормально падает плоская волна с частотой $\omega$, электрический вектор которой линейно поляризован вдоль оси $z$. Для феррита заданная поляризация электрического поля соответствует ТЕ волне с компонентами поля $\left(H_{x}, H_{y}, H_{z}\right)$ и эффективной МП вида

$$
\mu_{2}=\mu_{\perp}=\mu-\mu_{a}^{2} / \mu
$$

имеющей резонансный характер полевой и частотной зависимостей [17]. Дальнейший анализ проводился для следующих значений поля $H_{0}$ и частоты $f=\omega / 2 \pi$, отвечающих областям отрицательных значений $\operatorname{Re}\left(\mu_{\perp}\right)$ :

- на фиксированной частоте $f=2 \mathrm{GHz}$ при $H_{0}<H_{r}$, где $H_{r}=243 \mathrm{Oe}$ - поле ферромагнитного резонанса;

- при $H_{0}=100$ Ое в интервале частот $f_{r}<f<f_{a r}$, где $f_{r}=2.2 \mathrm{GHz}$ и $f_{a r}=5.9 \mathrm{GHz}$ - значения частот резонанса и антирезонанса соответственно.

Далее будем считать, что материал ENG-слоев 1 и 3 свободен от потерь $\left(\varepsilon_{1}, \mu_{1}\right.$ - вещественные величины). Для упрощения анализа будем полагать $\mu_{1}=1$.

\section{Анализ условия идеального туннелирования}

Полученное в работе [7] условие ИТ при нормальном падении электромагнитной волны на симметричный трехслойник вида $\mathrm{ENG}-\mathrm{MNG}-\mathrm{ENG}$ в отсутствие потерь имеет вид:

$$
\tanh \varphi_{2}=\frac{2\left|Z_{1}\right|\left|Z_{2}\right| \cdot \sinh 2 \varphi_{1}}{\left.\left.\eta\left|Z_{1}\right|^{2}-\left|Z_{2}\right|^{2}\right)+\left|Z_{1}\right|^{2}+\left|Z_{2}\right|^{2}\right) \cdot \cosh 2 \varphi_{1}}
$$

где введены обозначения $\varphi_{j}=k_{0}\left|n_{j}\right| d_{j}(j=1,2)-$ оптические толщины слоев, $k_{0}=\omega / c-$ волновое число в вакууме, $c$ - скорость света в вакууме, $\left|n_{1}\right|=\left(\left|\varepsilon_{1}\right| \mu_{1}\right)^{1 / 2}$ и $\left|n_{2}\right|=\left(\varepsilon_{2}\left|\mu_{2}\right|\right)^{1 / 2}$ - модули комплексных показателей преломления, а $\left|Z_{1}\right|=\left(\mu_{1} /\left|\varepsilon_{1}\right|\right)^{1 / 2}$ и $\left|Z_{2}\right|=\left(\left|\mu_{2}\right| / \varepsilon_{2}\right)^{1 / 2}-$ модули комплексных импедансов ENG- и MNG-слоев, $\eta=\left(1-\left|Z_{1}\right|^{2}\right) /\left(1+\left|Z_{1}\right|^{2}\right)$. (Для структуры без потерь показатели преломления $n_{1,2}$ и импедансы $Z_{1,2}$ будут чисто мнимыми.)

Нас будут интересовать ненулевые корни $\left|\mu_{2}\right|$ уравнения (3) при фиксированных значениях всех прочих входящих в него параметров. В предельных случаях оптически тонких $\left(\varphi_{1,2} \ll 1\right)$ и оптически толстых $\left(\varphi_{1,2} \rightarrow \infty\right)$ слоев приближенные значения корней можно получить аналитически.

В приближении оптически тонких слоев $1-3$ уравнение (3) имеет один ненулевой корень $\left|\mu_{2}^{(1)}\right|$, равный

$$
\left|\mu_{2}^{(1)}\right|=\mu_{1}\left(\xi / \xi_{\min }-1\right),
$$

где введен параметр $\xi=d_{1} / d_{2}$, равный отношению толщин ENG- и MNG-слоев, а $\xi_{\min }=0.5 \varepsilon_{2} /\left(\left|\varepsilon_{1}\right|+\mu_{1}\right)$. Видно, что корень $\left|\mu_{2}^{(1)}\right|$ существует только при достаточно больших значениях параметра $\xi>\xi_{\min }$ (при $\xi>\varepsilon_{2} / 2 \mu_{1}$ он будет существовать при любом значении $\left.\left|\varepsilon_{1}\right|\right)$.

Теперь рассмотрим случай оптически толстого ENG-слоя, т.е. $\varphi_{1} \rightarrow \infty$. Тогда $\sinh \varphi_{1} \approx \cosh \varphi_{1} \gg 1$ и (3) принимает вид

$$
\tanh \varphi_{2} \approx \frac{2\left|Z_{1}\right|\left|Z_{2}\right|}{\left|Z_{1}\right|^{2}+\left|Z_{2}\right|^{2}}
$$

Если предположить, что при этом MNG-слой является оптически тонким, то $\tanh \varphi_{2} \approx \varphi_{2}$ и можно найти корень $\left|\mu_{2}^{(1)}\right|$ :

$$
\left|\mu_{2}^{(1)}\right|=\left|Z_{1}\right|\left(\frac{2}{k_{0} d_{2}}-\varepsilon_{2}\left|Z_{1}\right|\right) .
$$

Если же слой 2 так же как и прилегающие слои 1 и 3, будет оптически толстым, то значение корня $\left|\mu_{2}^{(1)}\right|$ будет стремиться к величине

$$
\left|\mu_{2}^{(1)}\right|=\left(4 \mu_{1} \varepsilon_{2}\right) /\left|\varepsilon_{1}\right|,
$$

которая в отличие от предыдущего случа, не зависит от толщины слоя феррита.

На рис. 2 представлены результаты численного решения уравнения (3) для частоты $f=2 \mathrm{GHz}$. Зависимости корня $\left|\mu_{2}^{(1)}\right|$ от параметра $\xi$ на рис. 2, $a$ представлены для различных значений ДП при постоянном значении толщины MNG-слоя $d_{2}=0.2 \mathrm{~mm}$, а на рис. $2, b-$ 


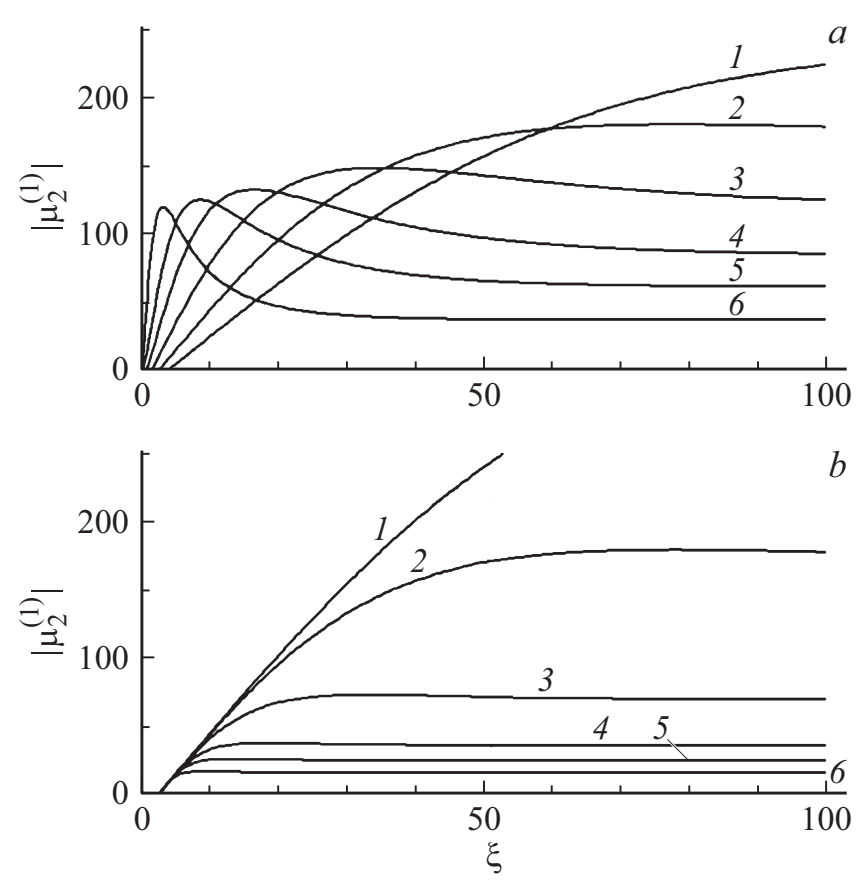

Рис. 2. Зависимости корня $\left|\mu_{2}^{(1)}\right|$ уравнения (3) от параметра $\xi=d_{1} / d_{2}$ на частоте $f=2 \mathrm{GHz}: a-d_{2}=0.2 \mathrm{~mm},\left|\varepsilon_{1}\right|=1,2$, $4,8,15,40$ (кривые $1-6$ ); $b-\left|\varepsilon_{1}\right|=2, d_{2}=0.1,0.2,0.5,1.0$, $1.5,2.5 \mathrm{~mm}$ (кривые $1-6$ ).

для различных $d_{2}$ при постоянной ДП $\left|\varepsilon_{1}\right|=2$. При малых $\xi$, близких к предельным значениям $\xi_{\min }$, слои структуры можно считать оптически тонкими, и зависимости $\left|\mu_{2}^{(1)}\right|$ являются практически линейными в полном соответствии с формулой (4). Для больших $\xi$ корень $\left|\mu_{2}^{(1)}\right|$ стремится к некоторму постоянному значению. Из рис. $2, b$ видно, что эта величина уменьшается по мере роста толщины $d_{2}$ (в соответствии с приближенной формулой (6)). Однако для больших толщин (кривые 5-7) эта величина стремится к некоторому предельному значению, что согласуется с формулой (7). Это говорит о том, что при соответствующих толщинах все слои структуры могут считаться оптически толстыми. При небольших толщинах среднего слоя $d_{2}$ кривые зависимостей $\left|\mu_{2}^{(1)}\right|(\xi)$ имеют характерный максимум, который виден на кривых 3-6 рис. 2, $a$.

Из проведенного анализа следует, что в случаях малых или больших оптических толщин $\varphi_{1,2}$ уравнение (3) имеет лишь один ненулевой корень. Однако численный анализ показал, что имеется область „промежуточных“ значений $\varphi_{1,2}$, где появляется еще один ненулевой корень $\left|\mu_{2}^{(2)}\right|<\left|\mu_{2}^{(1)}\right|$. Значения МП ферритового слоя, соответствующие корням уравнения (3), можно подобрать, изменяя подмагничивающее поле $H_{0}$ или частоту падающей волны $f$. При отсутствии потерь пропускательная способность структуры в этом случае достигала бы единицы. Далее приводятся результаты расчетов пропускательной способности структуры с учетом электрических и магнитных потерь в феррите.

\section{Расчет пропускательной способности структуры}

Считая волновые поля пропорциональными временному множителю $\exp (-i \omega t)$, запишем выражения для $z$-компоненты электрического поля волн во всех пространственных областях:

$$
E_{z}(x)= \begin{cases}\exp \left(i k_{0} x\right)+r \exp \left(-i k_{0} x\right), & x<0 \\ a_{1} \exp \left(i k_{1} x\right)+b_{1} \exp \left(-i k_{1} x\right), & 0<x<x_{1}, \\ a_{2} \exp \left(i k_{2} x\right)+b_{2} \exp \left(-i k_{2} x\right), & x_{1}<x<x_{2}, \\ a_{3} \exp \left(i k_{1} x\right)+b_{3} \exp \left(-i k_{1} x\right), & x_{2}<x<x_{3}, \\ t \exp \left(i k_{0} x\right), & x>x_{3},\end{cases}
$$

где $k_{j}=k_{0}\left(\varepsilon_{j} \mu_{j}\right)^{1 / 2}(j=1,2)$ - волновые числа в соответствующих слоях. Во всех областях с $x<x_{3}$ волновые поля являются суперпозицией полей двух встречных волн. В области $x>x_{3}$ существует только одна прошедшая через структуру волна. Согласно уравнениям Максвелла, поперечные компоненты магнитных волновых полей для вакуума определяются соотношениями

$$
H_{y}^{ \pm}=\mp E_{z}^{ \pm}
$$

а для MNG- и ENG-слоев

$$
H_{y}^{ \pm}=\mp Z_{j}^{-1} E_{z}^{ \pm},
$$

где $Z_{j}=\left(\mu_{j} / \varepsilon_{j}\right)^{1 / 2}-$ импедансы соответствующих сред, верхние знаки относятся к вперед бегущим волнам, нижние - к назад бегущим.

Приравнивая тангенциальные компоненты электрического и магнитного полей на границах раздела сред $x=0, x_{1}, x_{2}, x_{3}$, получаем систему уравнений:

$$
\begin{gathered}
1+r=a_{1}+b_{1}, \\
Z_{1}(-1+r)=-a_{1}+b_{1}, \\
a_{1} \exp \left(i k_{1} x_{1}\right)+b_{1} \exp \left(-i k_{1} x_{1}\right) \\
=a_{2} \exp \left(i k_{2} x_{1}\right)+b_{2} \exp \left(-i k_{2} x_{1}\right), \\
Z_{2}\left(-a_{1} \exp \left(i k_{1} x_{1}\right)+b_{1} \exp \left(-i k_{1} x_{1}\right)\right) \\
=Z_{1}\left(-a_{2} \exp \left(i k_{2} x_{1}\right)+b_{2} \exp \left(-i k_{2} x_{1}\right)\right), \\
a_{2} \exp \left(i k_{2} x_{2}\right)+b_{2} \exp \left(-i k_{2} x_{2}\right) \\
=a_{3} \exp \left(i k_{1} x_{2}\right)+b_{2} \exp \left(-i k_{1} x_{2}\right), \\
Z_{1}\left(-a_{1} \exp \left(i k_{2} x_{2}\right)+b_{1} \exp \left(-i k_{2} x_{2}\right)\right) \\
=Z_{2}\left(-a_{3} \exp \left(i k_{1} x_{2}\right)+b_{2} \exp \left(-i k_{1} x_{2}\right)\right), \\
a_{3} \exp \left(i k_{1} x_{3}\right)+b_{3} \exp \left(-i k_{1} x_{3}\right)=t \exp \left(i k_{0} x_{3}\right), \\
-a_{3} \exp \left(i k_{1} x_{3}\right)+b_{3} \exp \left(-i k_{1} x_{3}\right)=-Z_{1} t \exp \left(i k_{0} x_{3}\right),
\end{gathered}
$$


решение которой позволяет найти амплитудные коэффициенты $a_{j}, b_{j}$ для вперед и назад бегущих волн в каждом слое, а также амплитудные коэффициенты отражения $r$ и прохождения $t$. Пропускательная способность, т.е. энергетический коэффициент прохождения для структуры, расположенной между двумя одинаковыми средами, может быть найден далее как $T=|t|^{2}$.

\section{Численный анализ}

Ввиду сложности системы (11) ее решение проводилось численными методами. Вначале рассмотрим полевые зависимости пропускательной способности структуры $T$ на фиксированной частоте $f=2 \mathrm{GHz}$ в до-
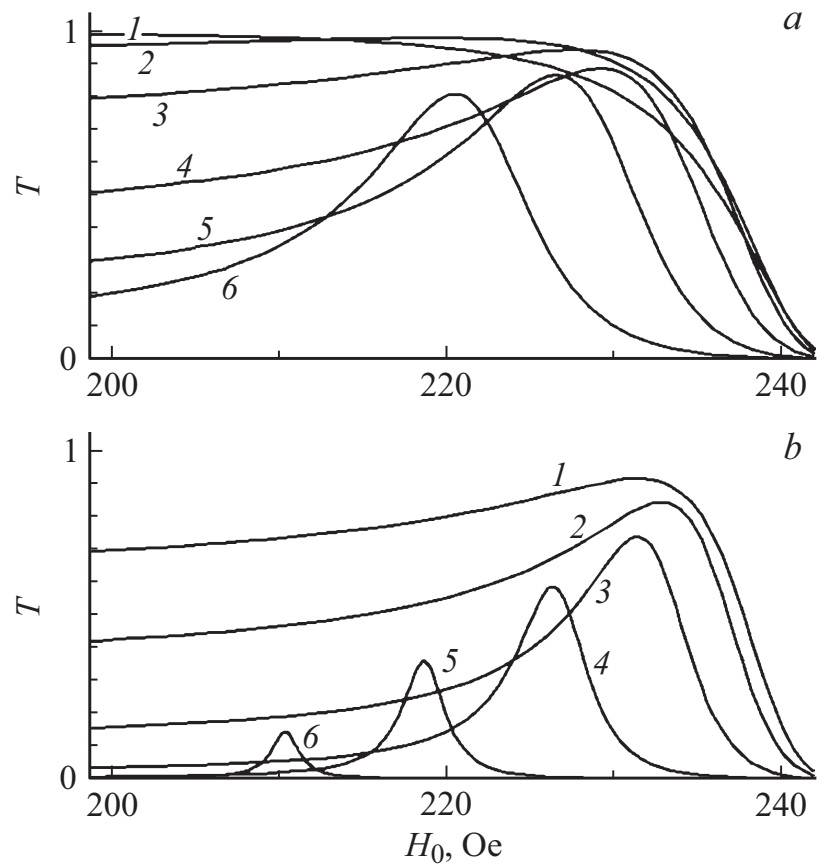

Рис. 3. Полевые завиисмости пропускательной способности структуры при $f=2 \mathrm{GHz}$ и $d_{2}=0.2 \mathrm{~mm}: a-\xi=10,\left|\varepsilon_{1}\right|=1$, $4,8,15,25,40$ (кривые $1-6$ ); $b-\xi=50,\left|\varepsilon_{1}\right|=1,2,4,8,15$, 25 (кривые $1-6$ ).

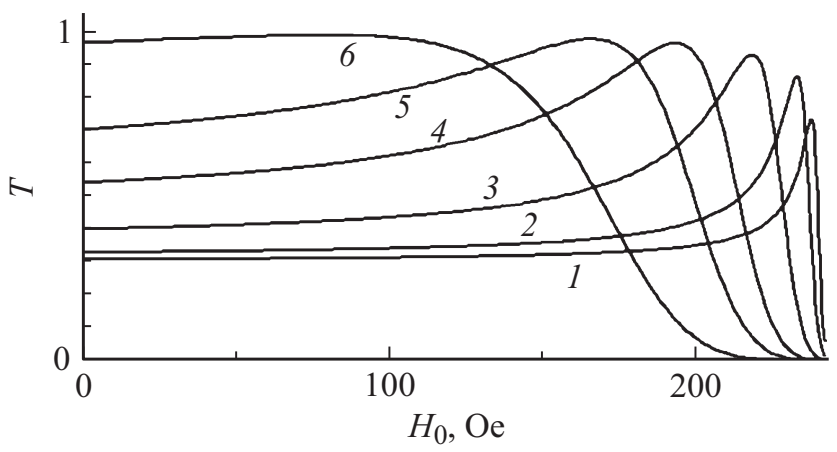

Рис. 4. Полевые завиисмости пропускательной способности структуры при $f=2 \mathrm{GHz}$ и $d_{1}=10 \mathrm{~mm},\left|\varepsilon_{1}\right|=2, d_{2}=0.1,0.2$, $0.5,1.0,1.5,2.5 \mathrm{~mm}$ (кривые 1-6).
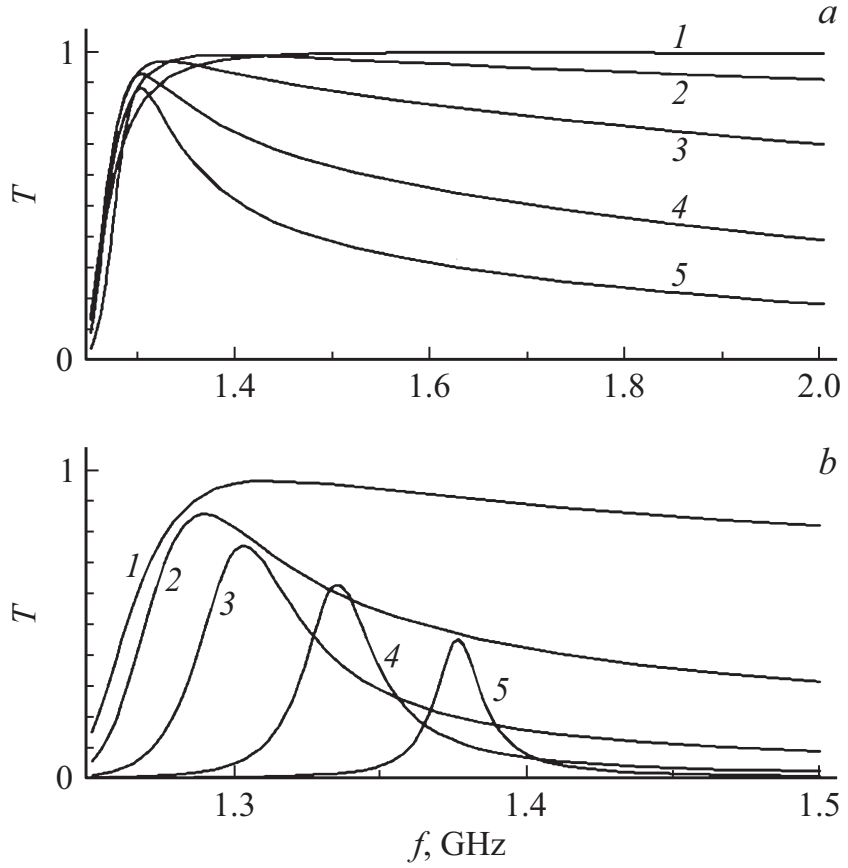

Рис. 5. Частотные завиисмости пропускательной способности структуры при $H_{0}=100 \mathrm{Oe}, d_{2}=0.2 \mathrm{~mm}, \xi=10(a)$ и $50(b)$, $\left|\varepsilon_{1}\right|=1,4,8,15,25$ (кривые $1-5$ ).

резонансной области полей, где $\operatorname{Re} \mu_{2}<0$. На рис. 3 представлены зависимости $T\left(H_{0}\right)$ для фиксированного значения толщины $d_{2}=0.2 \mathrm{~mm}$, значений параметpa $\xi=10,50(a, b)$ и различных значений ДП ENGслоя $\left|\varepsilon_{1}\right|$. Наблюдаемые пики пропускания становятся более ярко выраженными по мере увеличения оптических толщин слоев структуры. Пики при этом несимметричны: слева от пика величина $T$ слабо зависит от поля, а справа более резко спадает вследствие приближения к резонансу. Ввиду наличия максимумов у кривых $\left|\mu_{2}^{(1)}\right|(\xi)$ значения $\operatorname{Re} \mu_{2}$, дающие максимум пропускания, ограничены сверху. Поэтому по мере увеличения параметра $\left|\varepsilon_{1}\right|$ максимум пропускания сначала приближается к резонансному значению поля, а затем начинает удаляться от него. Заметим, что с увеличением $\left|\varepsilon_{1}\right|$, т.е. с ростом оптической толщины ENG-слоев $\varphi_{1}$ „пиковая“ величина пропускания $T_{\max }$ уменьшается (даже когда пик удаляется от резонанса).

Следовательно, для получения высоких значений $T$ пики пропускания должны располагаться как можно дальше от резонансного поля $H_{r}$, т. е. при относительно малых абсолютных значениях $\operatorname{Re} \mu_{2}$. При этом электрическая толщина слоев не должна быть слишком высокой. Эти условия могут быть выполнены в области малых оптических толщин, т.е. при значениях параметра $\xi$, близких к $\xi_{\text {min. }}$ Соответствующего уменьшения $\xi$ можно добиться, например, увеличением толщины слоя феррита $d_{2}$. На рис. 4 представлены зависимости $T\left(H_{0}\right)$ при фиксированных параметрах ENG-слоев $d_{1}=10 \mathrm{~mm}$ и $\left|\varepsilon_{1}\right|=2$ и различных значениях толщины феррита $d_{2}$. 

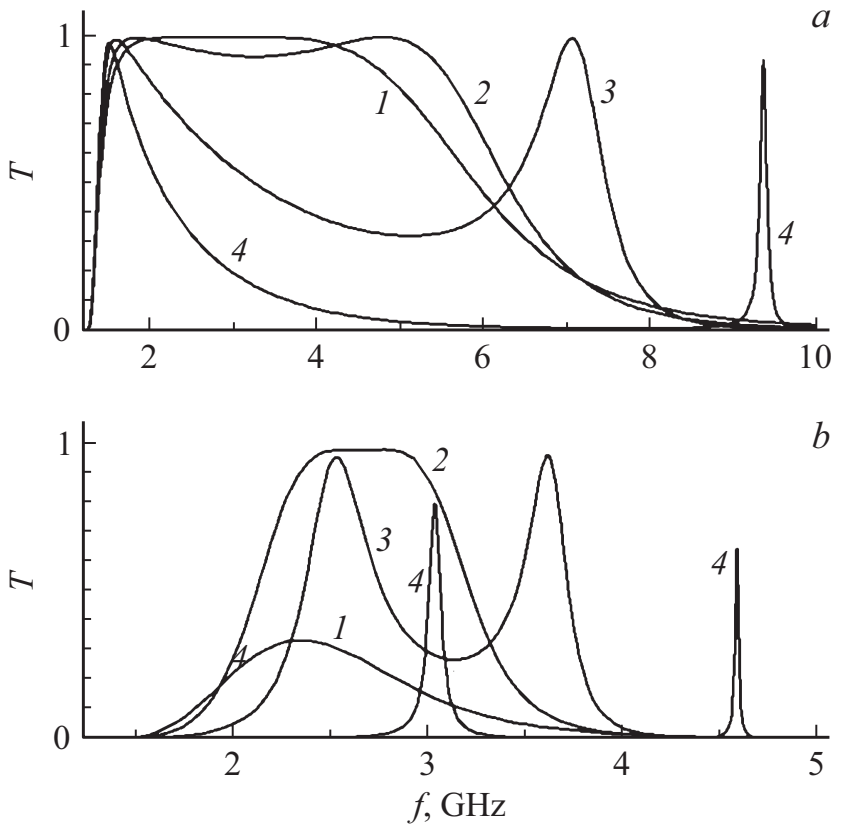

Рис. 6. Частотные завиисмости пропускательной способности структуры при $H_{0}=100 \mathrm{Oe}$ и $d_{1}=10 \mathrm{~mm}: a-d_{2}=1 \mathrm{~mm}$, $\left|\varepsilon_{1}\right|=0.15,0.5,1.25,2.25$ (кривые $1-4$ ); $b-d_{2}=5 \mathrm{~mm}$, $\left|\varepsilon_{1}\right|=2.0,3.8,5.5,10.0$ (кривые 1-4).

Видно, что с увеличением $d_{2}$ пики пропускания смещаются в сторону уменьшения поля $H_{0}$, а их высота при этом увеличивается. При $\xi \approx \xi_{\min }$ (кривая 6 ) достигается „почти идеальное“ туннелирование в широкой области полей шириной примерно 100 Ое. Однако при дальнейшем увеличении $d_{2}$ условие (3) перестает выполняться и пропускание в исследуемой области полей существенно уменьшается, становясь постепенно сравнимым с пропусканием одиночного слоя феррита.

Частотные зависимости пропускательной способности исследовались при фиксированном значении поля $H_{0}=100 \mathrm{Oе}$. На рис. 5 представлены зависимости $T(f)$ при сравнительно небольшом значении толщины феррита $d_{2}=0.2 \mathrm{~mm}$ для двух значений параметра $\xi=10,50(a, b)$ и различных значений $\left|\varepsilon_{1}\right|$. Особенности данных частотных зависимостей аналогичны представленным выше полевым зависимостям для структур с совпадающими параметрами (см. рис. 3). В частности, видно, что максимумы частотных зависимостей сгруппированы вблизи резонансной частоты.

На рис. 6 представлены частотные зависимости коэффициента пропускания $T(f)$ для структуры с $d_{1}=10 \mathrm{~mm} \quad$ и $d_{2}=1,5 \mathrm{~mm} \quad(a, b)$, полученные при различных значениях $\left|\varepsilon_{1}\right|$. В случае более тонкого MNG-слоя при значении $\left|\varepsilon_{1}\right| \approx 0.15$ (кривая 1) частотная зависимость пропускания имеет широкое „плато“ с практически постоянной величиной $T=0.99$, перекрывающее почти всю частотную область между резонансом и антирезонансом. Причина этого заключается в существовании при данной комбинации параметров двух корней уравнения (3). Плато образуется между двумя пиками пропускания, отвечающими корням $\left|\mu_{2}^{(1)}\right|$ и $\left|\mu_{2}^{(2)}\right|$, если они располагаются на достаточно близких частотах. По мере увеличения $\left|\varepsilon_{1}\right|$ пики все более четко разделяются (кривые $2-4$ ). Пик, соответствующий корню $\left|\mu_{2}^{(1)}\right|$, смещается слабо в сторону частоты $f$, а пик, соответствующий меньшему корню $\left|\mu_{2}^{(2)}\right|$, удаляется от резонанса и в итоге выходит за пределы области отрицательных значений $\operatorname{Re} \mu_{2}$. При $f>f_{a r}=5.9 \mathrm{GHz}$ $\operatorname{Re} \mu_{2}>0$ и исследуемый трехслойник представляет собой уже ENG-DPS-ENG-структуру. Аналогичные зависимости для структуры с большим значением толщины MNG-слоя показывают, что полоса пропускания (реализуемая при $\left|\varepsilon_{1}\right| \approx 3.8$ ) является более узкой, а ее левый край смещен от резонанса в сторону частоты $f_{a r}$.

В заключение отметим, что пропускательная способность исследуемой структуры легко управляема внешним магнитным полем. Так, с увеличением внешнего поля полоса пропускания структуры смещается по частоте в сторону ее увеличения, а ширина полосы уменьшается. При этом характер зависимостей, реализуемых при различных значениях толщины слоев и параметра $\left|\varepsilon_{1}\right|$, сохраняется с изменением подмагничивающего поля.

Работа выполнена при поддержке Министерства образования и науки РФ (государственное задание № 3.6825.2017 / БЧ и проект № 14.Z50.31.0015).

\section{Список литературы}

[1] Alù A., Engheta N. // IEEE Trans. on Antennas and Propag. 2003. Vol. 51. N 10. P. 2558-2571.

[2] Baena J.D., Jelinek L., Marqués R., Medina F. // Phys. Rev. E. 2003. Vol. 72. N 7. P. 075116.

[3] Zhou L., Wen W., Chan C.T., Sheng P. // Phys. Rev. Lett. 2005. Vol. 94. N 24. P. 243905.

[4] Marqués R., Martín F., Sorolla M. Metamaterials with negative parameters: theory, design, and microwave applications. NY.: John Wiley \& Sons, Inc., 2008. 309 p.

[5] Kim K.-Y., Lee B. // Phys. Rev. A. 2008. Vol. 77. N 2. P. 023822.

[6] Jelinek L., Baena J.D., Voves J., Marques R. // New J. Phys. 2011. Vol. 13. N 8. P. 083011.

[7] Cojocaru E. // Prog. Electromagn. Res. 2011. Vol. 113. P. 227-249.

[8] Zheng J., Chen Y., Chen Z., Wang X., Han P., Yong Z., Wang Y., Leung C.W., Soukoulis C.M. // Opt. Express. 2013. Vol. 21. N 14. P. 16742-16752.

[9] Castaldi G., Galdi V., Alù A., Engheta N. // J. Opt. Soc. Am. B. 2011. Vol. 28. N 10. P. 2362-2368.

[10] Liu C.-H., Behdad N. // Prog. Electromagn. Res. B. 2012. Vol. 42. P. 1-22.

[11] Sabah C., Tugrul Tastan H., Dincer F., Delihacioglu K., Karaaslan M., Unal E. // Prog. Electromagn. Res. 2013. Vol. 138. P. 293-306.

[12] Chen Y., Huang S., Yan X., Shi J. // Chinese Opt. Lett. 2014. Vol. 12. N 10. P. 101601. 
[13] Afanas'ev S.A., Sementsov D.I., Yakimov Y.V. // Opt. Comm. 2016. Vol. 369. P. 164-170.

[14] Афанасьев С.А., Семенщов Д.И., Фёдорова И.В. // ЖТФ. 2017. Т. 87. Вып. 12. С. 1849-1853. [Afanas'ev S.A., Sementsov D.I., Fedorova I.V. // Tech. Phys. 2017. Vol. 62. N 12. P. $1848-1852$.

[15] Яковлев Ю.М., Генделев С.Ш. Монокристаллы ферритов в радиоэлектронике. М.: Сов. радио, 1975. 232 с.

[16] Крупичка $C$. Физика ферритов и родственных им магнитных окислов. Т. 2 / Пер. с нем. под ред. А.С. Пахомова. М.: Мир, 1976. 504 с.

[17] Гуревич А.Г., Мелков Г.А. Магнитные колебания и волны. М.: Физматлит, 1994. 464 с. 